\title{
sgRNA Orientation
}

\section{Sequence}

\begin{tabular}{lll} 
A1 & fwd & CACCGCGAAGTCCGGCGGGTCCGAC \\
A1 & rev & AAACGTCGGACCCGCCGGACTTCGC \\
A2 & fwd & CACCGGAAACCGCAGAGGTCGCGAC \\
A2 & rev & AAACGTCGCGACCTCTGCGGTTCC \\
A3 & fwd & CACCGGGTCGGCGGCGATGGCCGTC \\
A3 & rev & AAACGACGGCCATCGCCGCCGACCC \\
A4 & fwd & CACCGGATGGTGCAGGAGCGCGAGA \\
A4 & rev & AAACTCTCGCGCTCCTGCACCATCC \\
\hline
\end{tabular}

Chromosome 17

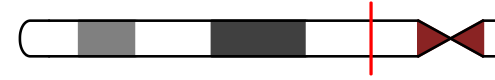

$19.55 \mathrm{mb}$

$19.57 \mathrm{mb}$

$19.56 \mathrm{mb}$

$19.58 \mathrm{mb}$

uc002gwb.1 다

uc002gwa.1 다)

uc010cqr.1

$\longrightarrow$

uc002gwd.1 다뇨

A1 \A4

A3 \A2

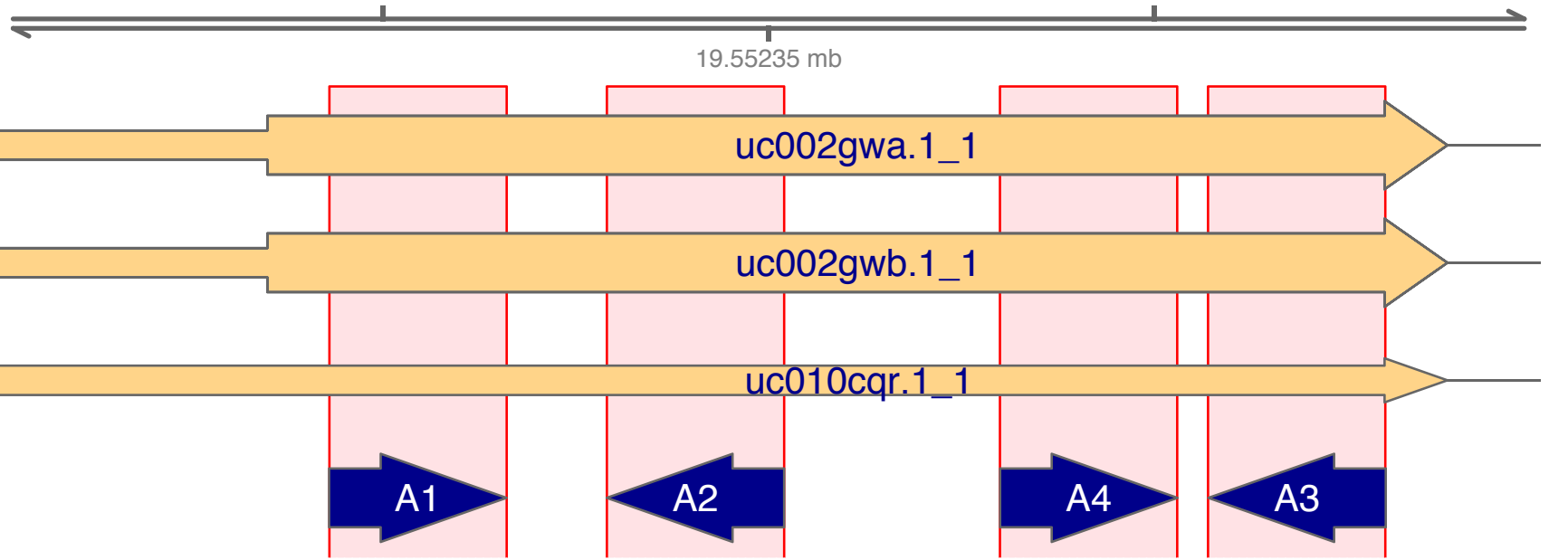

